\title{
BCAT1 promotes cell proliferation in aggressive gliomas
}

Expression of wild-type isocitrate dehydrogenase 1 (IDH1) or IDH2enzymes important for amino acid metabolism - in glioblastoma is a marker of poor prognosis, but the underlying mechanisms have been unclear. New research has highlighted a role for branched-chain amino acid transaminase 1 (BCAT1) in mediating cell proliferation of these aggressive brain tumours. As Bernhard Radlwimmer, who led the study, explains, "There appears to be a direct link between the function of IDH1 and BCAT1 in these tumours."

\section{4 ... a direct link [exists]} between the function of IDH1 and BCAT1 in these tumours 77

The researchers analysed the transcriptional profiles of astrocytic gliomas, and identified higher BCAT1 expression levels in gliomas with wild-type IDH1 compared with those expressing mutated IDH1. Methylation patterns of the BCAT1 gene promoter also differed between tumours with wild-type versus mutated IDH1, suggesting possible involvement of epigenetic regulation.

Cell proliferation in malignant cells is known to involve glutamine catabolism for fuel. BCAT1 catabolizes branched-chain amino acids (BCAAs) through transfer of an amino group to a-ketoglutarate (produced by IDH1 activity), yielding glutamate and branched-chain a-ketoacids.

The researchers showed that BCAT1 overexpression in glioma cell lines is dependent on the levels of a-ketoglutarate, and is suppressed by expression of mutant IDH1 in an astrocytic cell line. Production of $\alpha$-ketoglutarate by IDH1 might, therefore, drive BCAT1 overexpression in glioma.

Radlwimmer and colleagues found that inhibition of BCAT1 activity with a leucine analogue or RNA knockdown reduced tumour cell proliferation and glutamate release from these cells. Targeting BCAT1 activity in glioblastoma could, therefore, reduce neurotoxicity caused by excess glutamate release and help to avoid the occurrence of seizures and neurological dysfunction that are frequently associated with gliomas.

Next, the team investigated the role of BCAT1 in tumour growth in vivo. In mice transplanted with tumour cells, inhibition of BCAT1 activity significantly decreased tumour growth.

These findings suggest that targeting of BCAT1 could be a therapeutic approach to arrest tumour cell proliferation in aggressive glioma. As Radlwimmer points out, "Our study adds a new, so far underappreciated, aspect to tumour metabolism and potential metabolic reprogramming-BCAAs." The researchers plan to investigate BCAT1 and BCAA metabolism in other tumour types.

Ellen Bible

Original article Tonjes, M. et al. BCAT1 promotes cell proliferation through amino acid catabolism in gliomas carrying wild-type IDH1. Nat. Med. doi:10.1038/nm.3217 